\title{
Coronal Cavity Survey: Morphological Clues to Eruptive Magnetic Topologies
}

\author{
B.C. Forland · S.E. Gibson • J.B. Dove • \\ L.A. Rachmeler · Y. Fan
}

Received: 15 January 2013 / Accepted: 11 July 2013 / Published online: 3 September 2013

(C) The Author(s) 2013. This article is published with open access at Springerlink.com

\begin{abstract}
We present a survey on coronal prominence cavities conducted using 19 months of data from the Atmospheric Imaging Assembly (AIA) instrument aboard the Solar Dynamics Observatory (SDO) satellite. Coronal cavities are elliptical regions of rarefied density lying above and around prominences. They can be long-lived (weeks to months) but are often observed to eventually erupt as part of a coronal mass ejection (CME). We determine morphological properties of the cavities both by qualitatively assessing their shape, and quantitatively fitting them with ellipses. We demonstrate consistency between these two approaches, and find that fitted ellipses are taller than they are wide for almost all cavities studied, in agreement with an earlier analysis of white-light cavities. We examine correlations between cavity shape, aspect ratio, and propensity for eruption. We find that cavities with a teardrop-shaped morphology are more likely to erupt, and we discuss the implications
\end{abstract}

Coronal Magnetometry

Guest Editors: S. Tomczyk, J. Zhang, and T.S. Bastian

Electronic supplementary material The online version of this article

(doi:10.1007/s11207-013-0361-1) contains supplementary material, which is available to authorized users.

B.C. Forland · J.B. Dove

Physics Department, Metropolitan State University of Denver, Denver, CO 80217, USA

B.C. Forland

e-mail: bforland@msudenver.edu

B.C. Forland · S.E. Gibson ( $₫)$ · Y. Fan

High Altitude Observatory, NCAR, P.O. Box 3000, Boulder, CO 80307, USA

e-mail: sgibson@ucar.edu

B.C. Forland

e-mail: bforland@ucar.edu

Y. Fan

e-mail: yfan@ucar.edu

L.A. Rachmeler

University of St Andrews, St Andrews, Scotland, UK

e-mail: lar20@st-andrews.ac.uk 
of this morphology for magnetic topologies associated with CME models. We provide the full details of the survey for broad scientific use as supplemental material.

Keywords Solar corona $\cdot$ Magnetic fields $\cdot$ Prominences $\cdot$ Cavities $\cdot$ Coronal mass ejections

\section{Introduction}

Coronal cavities, commonly observed in association with prominences (also known as filaments), possess clearly defined boundaries implying a self-contained organized magnetic system. Cavities are one component of a characteristic three-part magnetic field structure observed in the corona: a central prominence, an elliptical region of depleted material that is often referred to as the cavity itself, and a surrounding bright plasma structure (e.g., arcade loops or a helmet streamer). Characterizing coronal magnetic structures is helpful for understanding the magnetic field of the corona as a whole; since cavities are often seen in association with coronal mass ejections (CMEs), a better understanding of their structure and evolution helps illuminate pre-CME magnetic field configurations (Engvold, 1989; Hudson et al., 1999; Low and Hundhausen, 1995; Gibson et al., 2006; Maričić, Vršnak, and Rosa, 2009; Régnier, Walsh, and Alexander, 2011).

Multiple approaches can be used to describe and infer information about the structure of the magnetic field of coronal cavities. One method is to make measurements sensitive to the coronal magnetic field. Using the Coronal Multi-channel Polarimeter (CoMP), it is possible to obtain information about the direction of the underlying magnetic fields (Tomczyk et al., 2008). This is complicated by the fact that the corona is optically thin; however, forward modeling of specific magnetic field topologies has been demonstrated as an effective means of enabling interpretation of the CoMP observations of cavities (Dove et al., 2011; Rachmeler, Casini, and Gibson, 2012; Rachmeler et al., 2013; Bạk-Stȩślicka et al., 2013).

The approach utilized in this paper is to analyze observational data from the Atmospheric Imaging Assembly (AIA: Lemen et al., 2012) on board the Solar Dynamics Observatory (SDO: Pesnell, Thompson, and Chamberlin, 2012), characterizing the morphology of cavities and determining any relationship they may have to eruptions. Our objective is to create a database of coronal cavities to identify morphologies indicative of pre-eruptive magnetic configuration and to also make the database available for future study. The high cadence of AIA and the fact that it has been operating for the rise of the solar cycle make the AIA dataset ideal for this type of analysis. Cavities are visible on a daily basis in this dataset and are clearly distinguishable from other structures in the corona - especially near the poles. We conduct a survey of 19 months of data and create a database that notes specific characteristics of the cavities, both qualitatively and quantitatively determined. We first establish a qualitative morphology of the observed cavities, classifying them as semicircular, elliptical, or teardrop-shaped. By fitting ellipses to each cavity, we are also able to quantify specific morphological properties such as cavity size, center position, and aspect ratio of ellipse width to height. We monitor the evolution of the cavity over several days, in order to identify a subset of cavities that erupt. In Section 2 we describe this methodology in detail. In Section 3, we present our analysis and results; in particular, our finding that both our qualitative assessment and quantitative measurements of cavity morphology indicate that a teardrop shape is an indicator of an increased propensity to erupt. In Section 4 we discuss these results in terms of theoretical models of magnetic precursors to CMEs, and in Section 5 we present our conclusions. 


\section{Methodology}

In order to determine which line to use for a survey of cavities, we examined the visibility of cavities in each of the SDO/AIA lines. Both the $211 \AA$ and $193 \AA$ lines were potential candidates: of these two lines we deemed $193 \AA$ more useful for our survey because it had a high contrast between the depleted region at the center of the cavity and the boundary between the cavity and the bright external plasma.

Although the $193 \AA$ line is the most suitable line, standard online images are not optimal for isolating cavities due to the steep intensity drop-off above the solar limb. For this reason we developed a new image processing routine. We fit the theta-averaged data from the solar limb to the edge of the AIA viewing area to a function we defined as

$$
I(r)=a \cdot \mathrm{e}^{-(r-1) / b},
$$

where $a$ and $b$ are fitting parameters and $I(r)$ is the intensity averaged over all polar angles for a given radius $r$. The intensity in each pixel above the limb is then multiplied by the inverse of $I(r)$. The result is an increase in the visibility and contrast of the cavities, making them more clear at higher heights (Figure 1).

We processed AIA data in this manner at a one-hour cadence to create movies covering the time interval 01 June 2010 through 31 December 2011. We then analyzed these movies in order to identify and characterize the cavities in our survey. Three-dimensional analyses of cavities indicate that they possess a tunnel-like morphology with an elliptical cross section (Gibson et al., 2010). Solar rotation of such a structure extended along the line of sight manifests as a two-dimensional, often elliptical, cavity at the solar limb lasting for several days but varying somewhat in size and shape.

In order to make our survey as reproducible and consistent as possible, we developed criteria for defining cavities. One of the fundamental cavity characteristics that we documented is the shape of the cavity. We categorized cavities as having a teardrop, elliptical, or semicircular shape (Figure 2).

Since the cavity is three dimensional, its two-dimensional projection could be described as one or more of these three shapes during its rotation past the limb. If a cavity was first observed as a semicircle but was later observed as an ellipse, it was recorded as an ellipse. If at any time a teardrop shape was observed, we classified that cavity as a teardrop.

We also determined criteria for labeling cavities in order to ensure that each observed structure was a unique cavity. Cavities often came into and out of visibility over the course of minutes or several hours. If a cavity reoccurred at the same latitude within 12 hours of a previously well-defined cavity, it was considered part of that same three-dimensional cavity structure. If more than 12 hours passed without a clear cavity, any cavity appearing at that latitude was classified as a separate structure. This meant that cavities were defined as distinct, although they may have been associated with the same extended filament channel. In addition, no attempts were made to connect cavities that existed on opposite limbs at the same latitude. Along with the beginning and end times and qualitative assessment of cavity morphology, many other characteristics of the cavities were documented. The full survey is available as supplemental online material. The entry for a single cavity is shown in Table 1 as an example of the information contained in the survey.

Of particular interest was that, for those cavities that were observed to erupt, we recorded information about the eruption such as the time of eruption, the point at which it was most clear that the cavity was unstable and that equilibrium had been lost, and the state of the cavity post-eruption. In associating a cavity with an eruption, in order to ensure that the 
Figure 1 Comparison of preand post-processed AIA $193 \AA$ Å images. (Top) Unprocessed image, (bottom) image processed with radial filter to highlight cavities above the limb. Note the increased visibility and detail of the cavity in the North-East associated with the extended northern polar-crown filament.

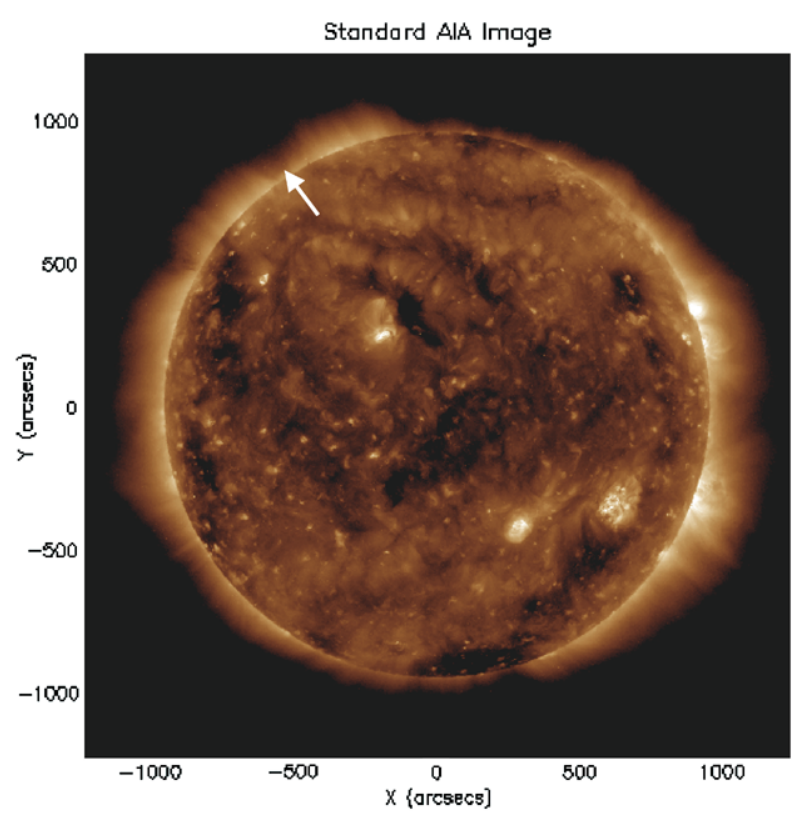

Fit Image

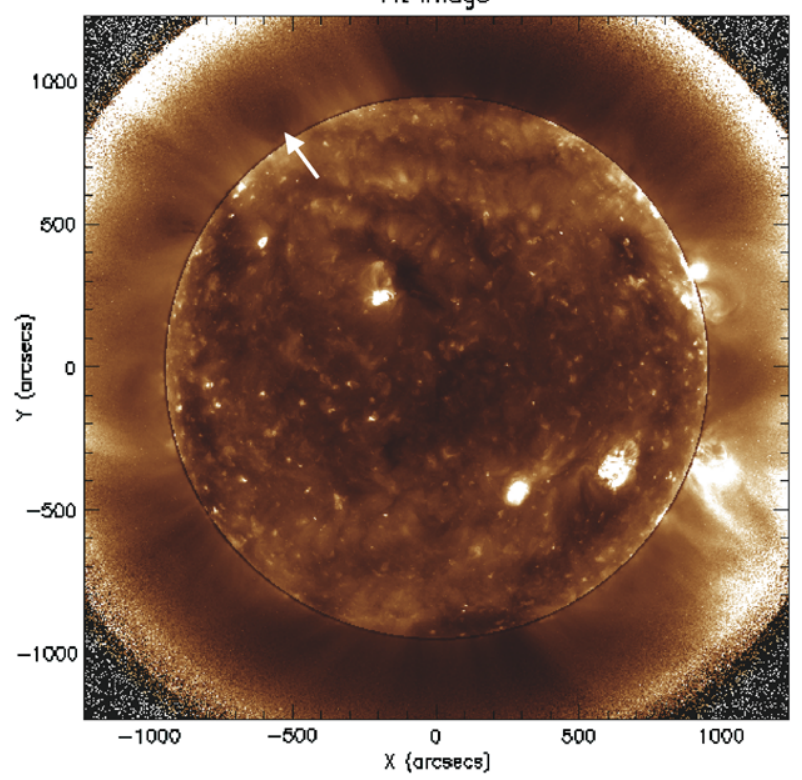

cavity existed before the eruption, we required the cavity to be clearly visible at the limb and stable (possessing nearly constant center height) for more than six hours prior to an eruption. In some cases an eruption occurred in the filament channel associated with a portion of the cavity that had rotated onto the disk. In such cases we required that some part of the cavity still be visible at the limb and that this limb portion be visibly perturbed by the eruption occurring in the filament channel. We noted cases in which there was an associated 

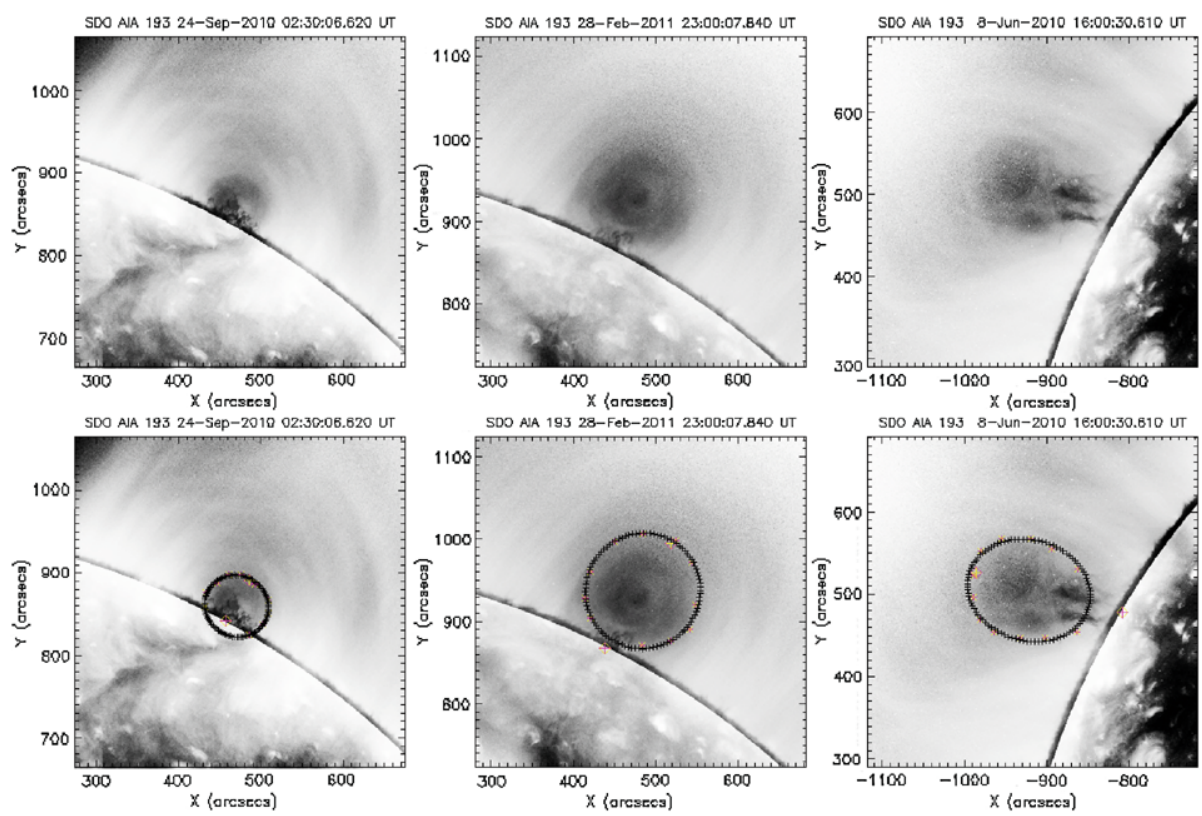

Figure 2 Examples of the three different morphologies used to characterize cavities for this survey. Semicircular (left) cavities did not show evidence of elliptical or oval cross section at any time while visible. Elliptical (middle) cavities possessed symmetry about both width and height axes. A teardrop shape (right) was assigned to cavities that departed from an elliptical shape (i.e., possessing a narrower bottom than top). Ellipses shown overlaid on bottom row are best fits to data - fit to semicircle extrapolates ellipse below the solar limb, and fit to teardrop is necessarily overwide at its base.

Table 1 Example survey entry for a coronal cavity. Quadrant of the cavity; Start and end times (when cavity was visible); Best time (when cavity was most visible - this is the time the cavity was fit with an ellipse); Shape (as described in text); Eruption time, type, and post-eruption status (as described in text); Axis height, colatitude (coordinates of center of ellipse fit); Aspect ratio (ratio of ellipse fit width to height, where height is axis closest to radial); Non-radial tilt (tilt of height axis from radial); Cavity bottom (radial height from photosphere of bottom of the ellipse, in tilted coordinate frame). The online supplemental table includes this information for all of the cavities and also the quantitative data from the ellipse fits that define the cavity size, position, and shape.

\begin{tabular}{llllll}
\hline Location & Start time & End time & Best time & Shape & $\begin{array}{l}\text { Eruption } \\
\text { (time) }\end{array}$ \\
\hline NW & $\begin{array}{l}2010-06-11 \\
10: 30: 08\end{array}$ & $\begin{array}{l}2010-06-13 \\
12: 30: 08\end{array}$ & $\begin{array}{l}2010-06-12 \\
19: 30: 08\end{array}$ & Teardrop & $\begin{array}{l}2010-06-13 \\
09: 30: 08\end{array}$ \\
\hline $\begin{array}{l}\text { Eruption } \\
\text { type }\end{array}$ & $\begin{array}{l}\text { Post- eruption } \\
\text { status }\end{array}$ & $\begin{array}{l}\text { Axis height } \\
R_{\text {Sun }}\end{array}$ & $\begin{array}{l}\text { Axis colatitude } \\
\text { Degrees }\end{array}$ & Aspect ratio & $\begin{array}{l}\text { Non-radial } \\
\text { tilt Degrees }\end{array}$ \\
\hline $\begin{array}{l}\text { Complete } \\
\text { cavity }\end{array}$ & $\begin{array}{l}\text { No clear cavity } \\
\text { visible }\end{array}$ & $1.20 \pm 0.011$ & $42 \pm 0.37$ (north) & $0.71 \pm 0.05$ & $-1.1 \pm 4.2$ \\
\hline
\end{tabular}

Ellipse

bottom

$R_{\text {Sun }}$

$1.02 \pm 0.01$ 
prominence eruption, and/or the cavity was observed to bodily erupt (Gibson et al., 2006; Maričić, Vršnak, and Rosa, 2009; Maričić et al., 2004).

In order to quantitatively analyze our cavities, we fitted them with ellipses (Figure 2, bottom row) and recorded information about their size, center position, and aspect ratio. This was done through a semi-interactive SolarSoft IDL program, in which users selected the border between the cavity region and the surrounding bright plasma to trace out the ellipse that best fit this boundary (Gibson et al., 2010). The program interpolated regions of ambiguity, such as those where the boundary could not be confidently traced, or where the boundary departed from an ellipse as in the case of teardrop-shaped cavities. The bottom of the ellipse could be either above or below the solar limb: semicircles were fit with ellipses that extended below the solar limb. Ellipse fitting of all the observed cavities was independently completed by three of the authors in order to gauge measurement error.

\section{Results}

We located 129 unique cavities in the 19 months of AIA data that we surveyed, classifying 39 teardrop-shaped cavities, 66 elliptical cavities, and 24 semicircular cavities. Of these 129 cavities, $28 \%$ erupted. $13 \%$ of semicircular and $17 \%$ of elliptical cavities, compared to $56 \%$ of teardrop-shaped cavities erupted. Teardrop-shaped cavities accounted for $61 \%$ of all eruptions observed (Figure 3, top).

We further considered observational biases associated with observing cavities at the east vs. the west limbs. Eruptions are as likely to occur on the back of the Sun as the front. As mentioned above, we examined the filament channel associated with the cavity for evidence of eruption. In several cases we identified front-side, east-limb eruptions where the cavity had almost completely rotated onto the disk and eruption would have been difficult to detect based solely on the remaining portion of the cavity at the limb. Equivalent cases where an eruption occurred after a west-limb cavity had largely rotated behind the west limb, were likely to go unnoticed. Such cases would therefore be "false negatives." (Similar east-limb, back-side and west-limb, front-side filament eruptions would not affect our survey since the eruptions would tend to remove the cavities before they could be identified.) Indeed, as indicated in Figure 3 (middle and bottom), we found $33 \%$ of east-limb cavities to be eruptive as opposed to $22 \%$ of west-limb cavities, indicative of our observational bias.

The associations with eruptions for the east limb are therefore more accurate, and Figure 3 (middle and bottom) shows that they strengthen the result that teardrop-shaped cavities are more likely to erupt: $68 \%$ of teardrop-shaped east-limb cavities erupted, as compared to $23 \%$ of elliptical and $10 \%$ of semicircular east-limb cavities. Teardrop-shaped cavities were thus nearly three times more likely to erupt than elliptical cavities, and seven times more likely to erupt than semicircular cavities.

The assignment of cavity shape as teardrop, elliptical, or semicircular was necessarily a qualitative assessment. We therefore turned to fits of the cavities to investigate the relationship between shape and eruption more quantitatively. We first considered the width-to-height aspect ratio of the ellipses that were fit to each cavity. Figure 4 shows that most cavities had aspect ratios less than one, implying a norm of "narrow" elliptical cavities. This is in agreement with an earlier survey of cavities observed in white light (Gibson et al., 2006), which found that cavities with heights less than 1.4 solar radii were taller than they were wide.

Figure 4 also demonstrates that cavities classified as teardrop-shaped had the smallest aspect ratios on average, followed by ellipses and then semicircles. Table 2 shows that this was true for all populations of cavities, including erupting and non-erupting. This indicates that 

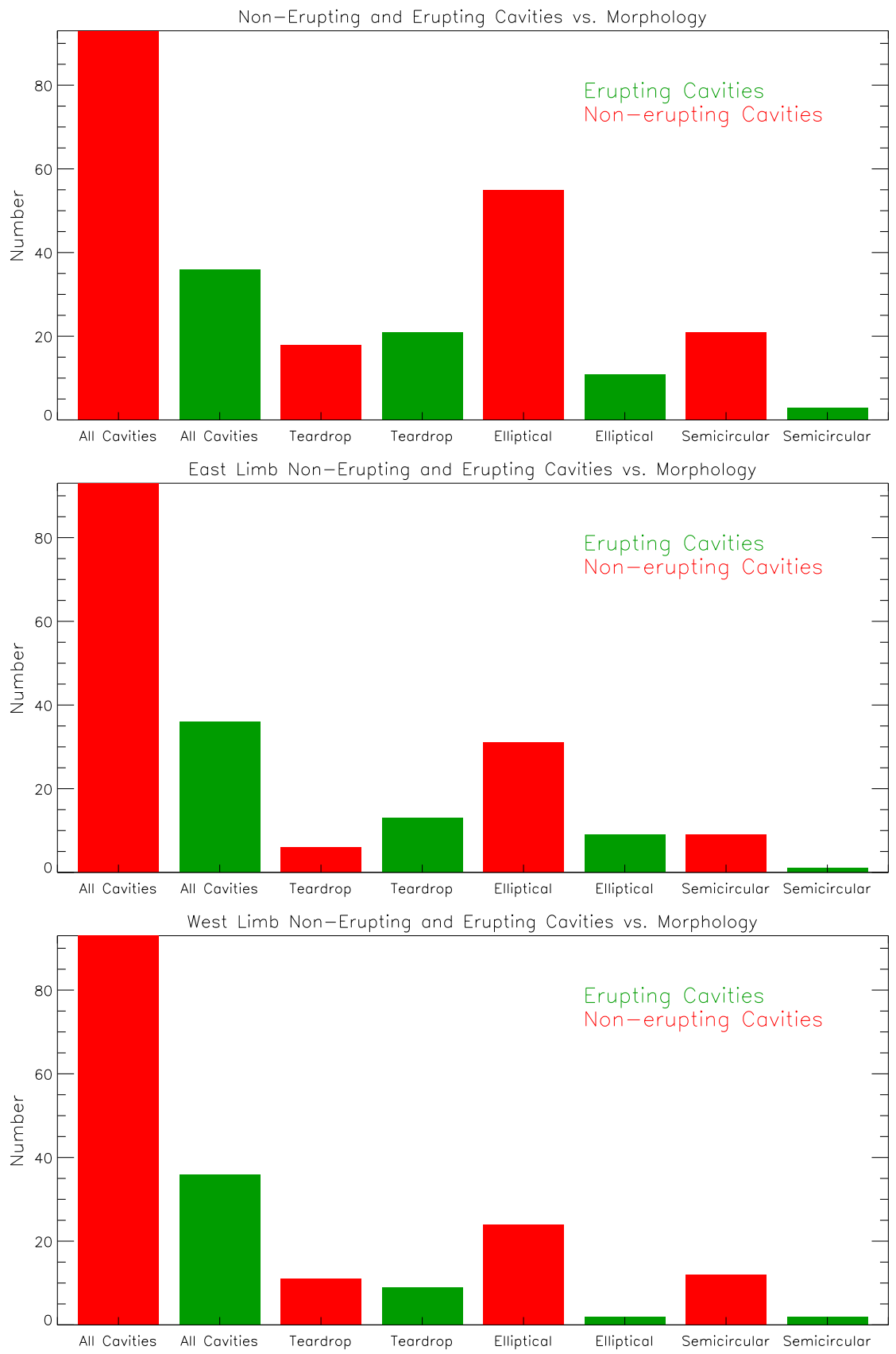

Figure 3 Comparison of the number of eruptive and non-eruptive cavities $v s$. morphology, with three samples: the full dataset, only east-limb cavities, and only west-limb cavities. Notice that not only do teardrop-shaped cavities have more eruptions than any other morphology, they are also the only morphology with more eruptive cases than non-eruptive cases. For reasons stated in the text, the east-limb results are most trustworthy for identification of eruptions; the predominance of erupting teardrop-shaped cavities is particularly clear for this subset. 


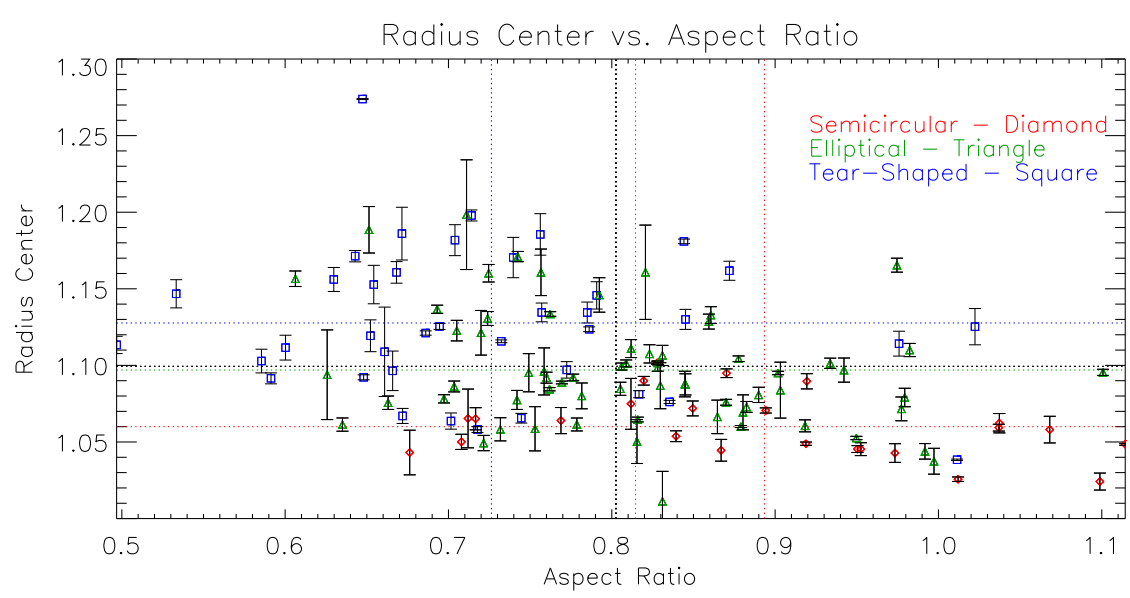

Cavity Bottom vs. Aspect Ratio

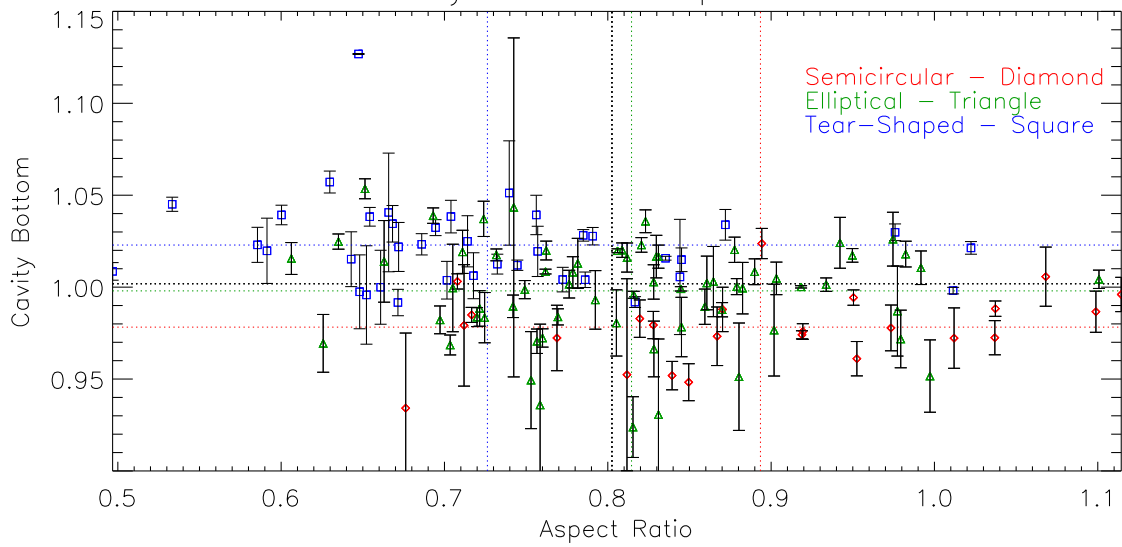

Figure 4 Cavity morphology plotted against ellipse parameters. (Top) Cavity center height (solar radii) $v s$. aspect ratio (width to height) shows a trend for teardrop-shaped cavities to possess smaller (narrower) aspect ratios and lie at higher heights. (Bottom) Height of cavity bottom (solar radii) vs. aspect ratio shows a related trend of teardrop-shaped cavities to be fit by ellipses lying completely above the limb. By definition, semicircular cavities were fit with ellipses with bottoms lying below the limb. Horizontal and vertical lines indicate averages for each of the populations, color-coded by morphology (black is the entire population). See also Table 2 .

our qualitative assessment of shapes is directly related to the quantitatively measured aspect ratio. However, the characterization of "teardrop-shaped" was not completely captured by the aspect ratio of our ellipses. Figure 4 (top) shows that the central height of the cavity is also correlated with its morphology; in particular, teardrop-shaped cavities on average had higher centers, followed by elliptical and then semicircular cavities. Figure 4 (bottom) demonstrates that teardrop-shaped cavities also tended to be fit to ellipses whose bottoms lay above the limb (by definition semicircular cavities were fit to ellipses with bottoms lying below the limb). As seen in Table 2, all three of these quantitatively measured properties aspect ratio, center height, and (to a lesser extent) bottom height - may be correlated to a teardrop-shaped morphology.

Figure 5 and Table 2 show that, consistent with our conclusion regarding teardrop-shaped cavities, on average eruptive cavities are narrow, high-centered, and high-bottomed. If we 
Table 2 Mean and standard deviation of properties of the cavity ellipse fits as described in Table 1 for various populations of cavities. East-limb cavities only are used for the non-erupting cases, because of the possibility of false negatives in west-limb analysis (see text).

\begin{tabular}{llll}
\hline & Mean aspect ratio & Mean axis height $R_{\odot}$ & $\begin{array}{c}\text { Mean ellipse } \\
\text { bottom } R_{\odot}\end{array}$ \\
\hline All tear-shaped & $0.73 \pm 0.12$ & $1.13 \pm 0.047$ & $1.02 \pm 0.024$ \\
All elliptical & $0.81 \pm 0.10$ & $1.10 \pm 0.037$ & $1.00 \pm 0.027$ \\
All semicircular & $0.89 \pm 0.13$ & $1.06 \pm 0.020$ & $0.98 \pm 0.020$ \\
All erupting & $0.73 \pm 0.13$ & $1.12 \pm 0.050$ & $1.01 \pm 0.036$ \\
Erupting tear-shaped & $0.70 \pm 0.11$ & $1.15 \pm 0.051$ & $1.03 \pm 0.028$ \\
Erupting elliptical & $0.78 \pm 0.12$ & $1.09 \pm 0.049$ & $0.99 \pm 0.039$ \\
Erupting semicircular & $0.86 \pm 0.16$ & $1.06 \pm 0.038$ & $0.98 \pm 0.020$ \\
All non-erupting & $0.83 \pm 0.12$ & $1.09 \pm 0.038$ & $1.00 \pm 0.025$ \\
Non-erupting tear-shaped & $0.76 \pm 0.12$ & $1.11 \pm 0.028$ & $1.02 \pm 0.017$ \\
Non-erupting elliptical & $0.82 \pm 0.10$ & $1.10 \pm 0.050$ & $1.00 \pm 0.023$ \\
Non-erupting semicircular & $0.90 \pm 0.13$ & $1.06 \pm 0.037$ & $0.98 \pm 0.021$ \\
\hline
\end{tabular}

take as a dividing line an aspect ratio of 0.8 , which is the average aspect ratio for the total population of cavities, we find that $40 \%$ of cavities with aspect ratios smaller than this (narrow cavities) erupt, as compared to $16 \%$ for the cavities with aspect ratios greater than this average. If we consider just east-limb cavities, we find the following: narrow cavities erupt $50 \%$ of the time; cavities with heights greater than average (1.1 solar radius) erupt $47 \%$ of the time (as opposed to $22 \%$ for cavities with heights below average); cavities with bottoms higher than average (1.0 solar radius) erupt $39 \%$ of the time (compared to $27 \%$ for cavities with bottoms below average). East-limb cavities possessing all three properties together - narrow aspect ratio, high center, and high bottom - erupt $77 \%$ of the time, as opposed to $20 \%$ for cavities with below-average values for these three qualities.

It is important to note that there is a significant spread in the ellipse properties of eruptive and non-eruptive cavities, as seen in the large standard deviations in the distributions (e.g., uncertainties). Figure 6 illustrates this for aspect ratio.

The cavities have been separated into eruptive (green) and non-eruptive (red) populations, and plotted as normal distributions for aspect ratio, with shading indicating one standard deviation on either side of average (see also Table 2). These standard deviations are approximately equivalent to the difference between the peaks in the normal distributions. This large spread is partly due to uncertainties in the fitting process, but may also be affected by both false positives and false negatives in identifying eruptive cavities in relation to aspect ratio. False negatives have been discussed above, and we have addressed them to some extent by plotting only east-limb cases in our baseline non-eruptive (red) distribution. False positives occur if a CME is associated with a cavity that has a relatively large aspect ratio, but the true aspect ratio has been misidentified because the cavity has not rotated fully into view before the eruption. It is also possible that some CMEs are triggered by a global redistribution of coronal magnetic fields which perturbs the cavity (Schrijver and Title, 2011), resulting in the loss of equilibrium of a large aspect-ratio cavity that otherwise would not have erupted. Despite these caveats, the consistency of our results, both qualitative and quantitative, leads us to believe that a teardrop-shaped morphology is a significant indicator of impending eruption. 


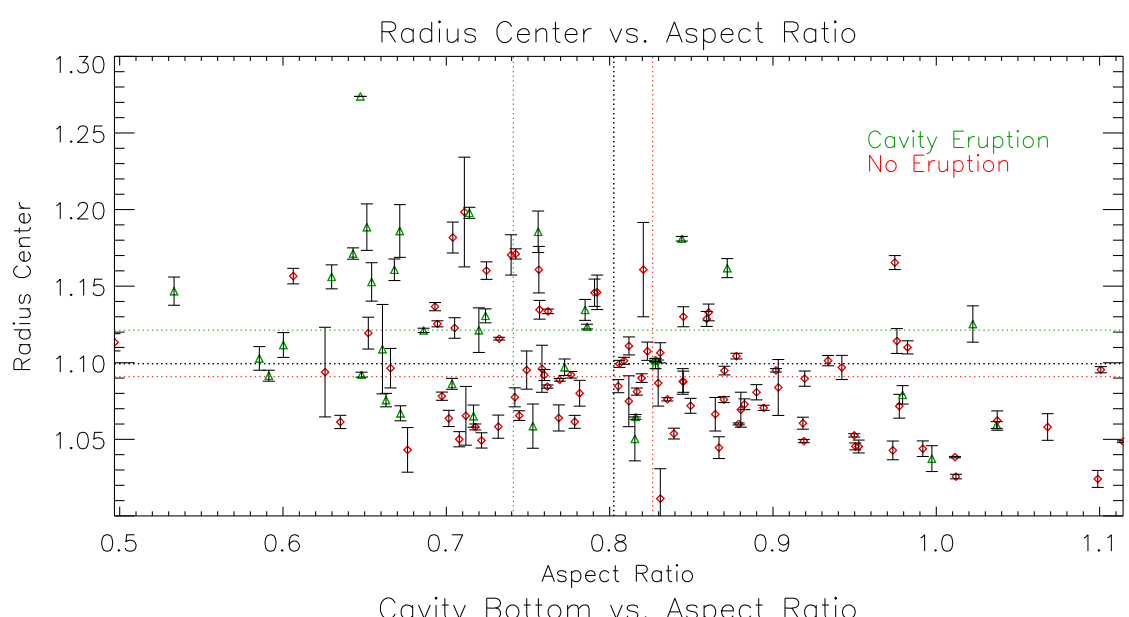

Cavity Bottom vs. Aspect Ratio

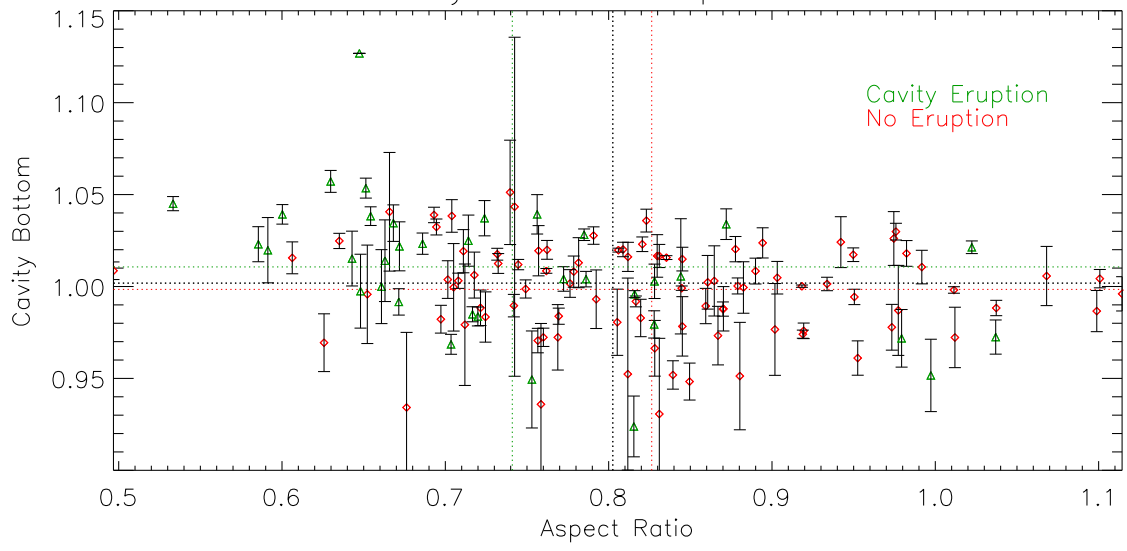

Figure 5 Eruptivity plotted against ellipse parameters. (Top) Cavity center height (solar radii) vs. aspect ratio (width to height) shows a trend for narrower, higher cavities to erupt. (Bottom) Height of cavity bottom (solar radii) $v s$. aspect ratio shows a related trend for those with higher bottom heights to erupt. Horizontal and vertical lines indicate averages for each of the populations, color-coded by eruptivity (black is the entire population). See also Table 2.

\section{Discussion}

The long life of the cavity before eruption indicates that it exists in a quasi-stable magnetohydrodynamic (MHD) equilibrium. The elliptical cross section of most cavities coupled with coronal magnetic observations of a sheared field at the heart of the cavity (Ba̧k-Stęślicka et al., 2013) suggest a magnetic flux rope topology. Loss of equilibrium of flux ropes has been simulated for cases where an ideal "torus instability" occurs due to the flux rope axis height lying above a critical point relative to the radial drop-off of the overlying field (Bateman, 1978; Török and Kliem, 2005; Fan, 2005). Recent simulations (Aulanier et al., 2010; Fan, 2010) find a slow evolution of the flux rope via reconnections that increase its magnetic helicity and its axis height to a point where it is unstable to the torus instability. The mechanisms leading up to the loss equilibrium may involve multiple processes (see Forbes and Isenberg, 1991; Vršnak, 1990; Török and Kliem, 2005). This hypothesis is supported by three-dimensional nonlinear force-free extrapolations of vector magnetic field observa- 


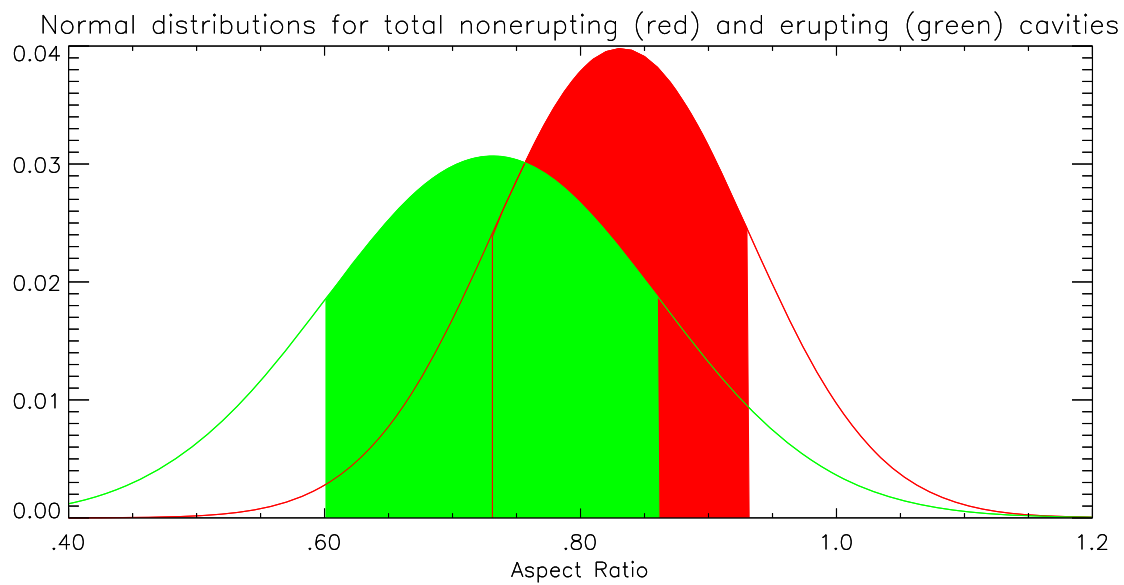

Figure 6 A normal distribution of the aspect ratio for total eruptive and east-limb non-eruptive cavities. The shaded region is an indication of one standard deviation up and down from the mean aspect ratio.
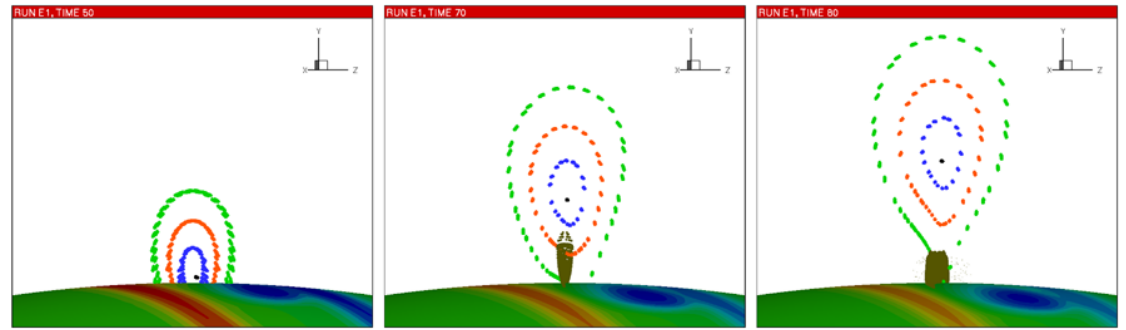

Figure 7 Magnetic flux surfaces (blue, orange, green) within a flux rope at three stages of evolution. Flux surfaces are shown in a plane perpendicular to the underlying polarity inversion line. The possible location of prominence mass accumulation is indicated by brown dots, which fill dips in magnetic field lines up to a prominence scale height $\left(0.01 R_{\odot}\right)$. (Left) The flux rope axis (black) is very low lying, and the flux surfaces are largely semicircular; (middle) flux surfaces are now elliptical, and dipped fields are now present; (right) the flux surfaces are now teardrop-shaped, and a current sheet is beginning to form aligned with the dipped magnetic field at the flux rope base. Compare to the three types of cavities in Figure 2.

tions, indicating the gradual formation of magnetic flux ropes and their eventual eruption (Savcheva et al., 2012). In that analysis, the flux ropes are initially confined to lower heights, but just before eruption they develop a teardrop-shaped cross section with vertical current sheets beneath the rising flux ropes.

Figure 7 illustrates a similar sequence of quasi-stable equilibrium from the flux rope simulation of Fan (2010). Initially, the amount of magnetic twist is minor, lying within semicircular flux surfaces of sheared field (Figure 7, left). After the flux rope axis emerges, the flux surfaces are ellipses. These ellipses are narrow, due to the upward expansion of the flux rope into the corona and lateral confinement by a surrounding arcade field. When helicity is further increased, a current sheet begins to form at the base of the flux rope aligned with the dipped field region identified with the filament, and the flux surfaces are teardrop-shaped (Figure 7, right).

If cavities are indeed signatures of magnetic flux ropes, we can explain the two main observational findings of this paper: the narrow elliptical cross section of cavities in general, 
and the association of a teardrop shape with impending eruption. The increased height of near-eruptive cavities is also consistent with the torus instability as a driver of eruption, and in general with theories of critical heights for eruption onset (Vršnak, Ruždjak, and Rompolt, 1991; Chen et al., 2006; Filippov and Koutchmy, 2008). This is related to prominence observations that indicate an upper limit for prominence height (Liu and Schuck, 2012), although we note that, as seen in Figure 7 (right), because of the curvature of the rope, the location of the prominence may lie well below its axis. It is possible that the center of the cavity is a better indicator of the axis than the prominence height. This height could be monitored to establish thresholds for the torus instability by comparisons to the coronal field (de Toma, private communication).

\section{Conclusions}

We have undertaken the first large survey of extreme ultraviolet coronal prominence cavities and have made the survey open and available for future research. Our analysis demonstrated connections between cavity morphology and eruptivity. Our two major findings are that cavities are nearly always narrow (with a larger height than width) and that teardropshaped cavities have an increased propensity for eruption. We have found by fitting ellipses to cavities that this teardrop-shaped morphology is correlated to some extent to aspect ratio (narrow), center height (high), and bottom height of ellipse (above limb), and that all of these quantities are associated with cavities that erupt from hours to days later. However, we believe that the fundamental quantity is the teardrop shape, and that it is indicative of topological changes associated with the formation of a current sheet beneath a flux rope. This acts as a slow-burning fuse, pushing the flux rope ever higher until ultimately it crosses a threshold height for the torus instability, and the cavity and some or all of its entrained prominence erupt as a coronal mass ejection.

Acknowledgements We would like to thank Giuliana De Toma, Don Schmit, Erin Wood, and the University of Colorado's Laboratory for Atmospheric and Space Physics (LASP) Research Experience for Undergraduates (REU) program. The National Center for Atmospheric Research (NCAR) is sponsored by the National Science Foundation. The AIA data was provided by the Joint Science Operations Center (JSOC) HMI-AIA Science Data Processing (SDP) home at Stanford University. This work is supported in part by the NASA LWS Grant NNX09AJ89G.

Open Access This article is distributed under the terms of the Creative Commons Attribution License which permits any use, distribution, and reproduction in any medium, provided the original author(s) and the source are credited.

\section{References}

Aulanier, G., Török, T., Démoulin, P., DeLuca, E.E.: 2010, Formation of torus-unstable flux ropes and electric currents in erupting sigmoids. Astrophys. J. 708, 314.

Ba̧k-Stȩślicka, U., Gibson, S.E., Fan, Y., Bethge, C., Forland, B., Rachmeler, L.A.: 2013, Twisted magnetic structure of solar prominence cavities: new observational signature revealed by coronal magnetometry. Astrophys. J. Lett. 770, L28. doi:10.1088/2041-8205/770/2/L28.

Bateman, G.: 1978, MHD Instabilities, MIT Press, Cambridge, 84-85.

Chen, J., Marqué, C., Vourlidas, A. Krall, J., Schuck, P.W.: 2006, The flux-rope scaling of the acceleration of CMEs and eruptive prominences. Astrophys. J. 649, 452.

Dove, J.B., Gibson, S.E., Rachmeler, L.A., Tomczyk, S., Judge, P.: 2011, A ring of polarized light: evidence for twisted coronal magnetism in cavities. Astrophys. J. 731, 1. 
Engvold, O.: 1989, In: Priest, E.R. (ed.) Dynamics and Structures of Quiescent Prominences, Reidel, Dordrecht, 47.

Fan, Y.: 2005, Coronal mass ejections as loss of confinement of kinked magnetic flux ropes. Astrophys. J. 630, 543.

Fan, Y.: 2010, On the eruption of coronal flux ropes. Astrophys. J. 719, 728.

Filippov, B., Koutchmy, S.: 2008, Causal relationships between eruptive prominences and coronal mass ejections. Ann. Geophys. 26, 3025 - 3031. doi:10.5194/angeo-26-3025-2008.

Forbes, T.G., Isenberg, P.A.: 1991, A catastrophe mechanism for coronal mass ejections. Astrophys. J. 373, 294. doi:10.1086/170051.

Gibson, S.E., Foster, D., Burkepile, J., de Toma, G., Stanger, A.: 2006, The calm before the storm: the link between quiescent cavities and CMEs. Astrophys. J. 641, 590.

Gibson, S.E., Kucera, T.A., Rastawicki, D., Dove, J., de Toma, G., Hao, J., Hill, S., Hudson, H.S., Marque, C., McIntosh, P.S., Rachmeler, L., Reeves, K.K., Schmieder, B., Schmit, D.J., Seaton, D.B., Sterling, A.C., Tripathi, D., Williams, D.R., Zhang, M.: 2010, Three-dimensional morphology of a coronal prominence cavity. Astrophys. J. 723, 1133.

Hudson, H.S., Acton, L.W., Harvey, K.A., McKenzie, D.M.: 1999, A stable filament cavity with a hot core. Astrophys. J. 513, 83.

Lemen, J.R., Title, A.M., Akin, D.J., Boerner, P.F., Chou, C., Drake, J.F., et al.: 2012, The Atmospheric Imaging Assembly (AIA) on the Solar Dynamics Observatory (SDO). Solar Phys. $275,17$. doi:10.1007/s11207-011-9776-8.

Liu, Y., Schuck, P.W.: 2012, Magnetic energy and helicity in two emerging active regions in the Sun. Astrophys. J. 761, 105. doi:10.1088/0004-637X/761/2/105.

Low, B.C., Hundhausen, J.R.: 1995, Magnetostatic structures of the solar corona. II. The magnetic topology of quiescent prominences. Astrophys. J. 443, 818.

Maričić, D., Vršnak, B., Rosa, D.: 2009, Relative kinematics of the leading edge and the prominence in coronal mass ejections. Solar Phys. 260, 177.

Maričić, D., Vršnak, B., Stanger, A.L., Veronig, A.: 2004, Coronal mass ejection of 15 May 2001: I. Evolution of morphological features of the eruption. Solar Phys. 225, 337.

Pesnell, W.D., Thompson, B.J., Chamberlin, P.C.: 2012, The Solar Dynamics Observatory (SDO). Solar Phys. 275, 3 - 15. doi:10.1007/s11207-011-9841-3.

Rachmeler, L.A., Casini, R., Gibson, S.E.: 2012, Interpreting coronal polarization observations. In: Rimmele, T.R., Tritschler, A., Wöger, F., Collados Vera, M., Socas-Navarro, H., Schlichenmaier, R., Carlsson, M., Berger, T., Cadavid, A., Gilbert, P.R., Goode, P.R., Knölker, M. (eds.) Astron. Soc. Pac. CS-463, 227.

Rachmeler, L.A., Gibson, S.E., Dove, J.B., DeVore, C.R., Fan, Y.: 2013, Polarimetric properties of flux ropes and sheared arcades in coronal prominence cavities. Solar Phys., in this issue. doi:10.1007/s11207013-0325-5.

Régnier, S., Walsh, R.W., Alexander, C.E.: 2011, A new look at a polar crown cavity as observed by SDO/AIA. Structure and dynamics. Astron. Astrophys. 533, L1. doi:10.1051/0004-6361/201117381.

Savcheva, A., Pariat, E., van Ballegooijen, A., Aulanier, G., DeLuca, E.: 2012, Sigmoidal active region on the sun: comparison of a magnetohydrodynamical simulation and a nonlinear force-free field model. Astrophys. J. 750, 15. doi:10.1088/0004-637X/750/1/15.

Schrijver, C.J., Title, A.M.: 2011, Long-range magnetic couplings between solar flares and coronal mass ejections observed by SDO and STEREO. J. Geophys. Res. 116(15), 4108. doi:10.1029/2010JA016224.

Tomczyk, S., Card, G.L., Darnell, T., Elmore, D.F., Lull, R., Nelson, P.G., Streander, K.V., Burkepile, J., Casini, R., Judge, P.G.: 2008, An instrument to measure coronal emission line polarization. Solar Phys. 247, 411.

Török, T., Kliem, B.: 2005, Confined and ejective eruptions of kink-unstable flux ropes. Astrophys. J. Lett. 630, L97.

Vršnak, B.: 1990, Eruptive instability of cylindrical prominences. Solar Phys. 129, 295. doi:10.1007/ BF00159042.

Vršnak, B., Ruždjak, V., Rompolt, B.: 1991, Stability of prominences exposing helical-like patterns. Solar Phys. 136, 151. doi:10.1007/BF00151701. 